\title{
Performance analysis of IEEE 802.11n under different STBC rates using 64-QAM
}

\author{
Anastasios G. Gravalos, Marios G. Hadjinicolaou and Qiang Ni \\ Electronic and Computer Engineering \\ School of Engineering and Design, Brunel University \\ Uxbridge, Middlesex, UB8 3PH, UK
}

\begin{abstract}
The main focus of current research and development for the next-generation wireless local area network (WLAN) communication systems is to enhance the link throughput and channel capacity. In this paper, the performance analysis of the ongoing next-generation WLAN standard, IEEE 802.11n high throughput WLAN PHY layer is presented. The design criteria is based on a $4 \times 4$ MIMO-OFDM scheme using 64-QAM (Quadrature Amplitude Modulation) technique under Rayleigh frequency-selective fading and flat fading channels. Simulation results show that a significant performance gain, 4 7 dB, depending on the channel selection, can be achieved when $1 / 2$ space-time block code (STBC) rate is applied with respect to bit-error-rate (BER). Furthermore, performance gain of $7 \mathrm{~dB}$ is achieved when the $4 \times 4$ system using $1 / 2 \mathrm{STBC}$ is compared with a $2 \times 2$ system using STBC code rate 1.
\end{abstract}

\section{Index Terms - MIMO, OFDM, STBC, WLAN, 802.11n.}

\section{INTRODUCTION}

$\mathrm{O}$ rthogonal Frequency Division Multiplexing (OFDM) is a multi-carrier transmission technique employed to reduce the intersymbol interference (ISI) of wireless systems with small symbol periods. As a result, the capacity in wireless systems is improved. Another promising transmission technique is multiple-input multiple-output (MIMO) which enables the enhancement of capacity and performance with low BER proportional to the number of antennas. Therefore high spectrally efficient wireless transmissions can be produced by using multiple transmit and multiple receive antennas. Further, the combination of MIMO and OFDM greatly reduces the equalization complexity [1].

The definition of MIMO systems is that with a given arbitrary wireless communication system, there is a link where the transmitting end and the receiving end are equipped with multiple antenna elements. The signals produced by both types of antennas combine in such a way, which result in a reduced BER or increased data rate (bits/sec). A major advantage of MIMO system is their ability to utilize multi-path propagation to increase the transmission efficiency and channel capacity by the use of random fading and multipath delay spread. In this way, many orders of magnitude enhancement can be achieved in wireless communications without the use of extra spectrum
[2]. The destructive addition of multipath in a propagation medium and the interference of other users results in severe attenuation of the transmitted signal over the wireless channel. The reliability of wireless communications is the product of diversity techniques such as temporal diversity, frequency diversity or antenna diversity [3].

In order to achieve the full diversity promised by transmit and received antennas, space time block code (STBC) is used [6]. The employment of more than one antenna at the transmitter and the proper coding of data across the transmit antennas physical layers (PHYs) with STBC guarantee an increased data rate with minimal decoding complexity at the receiver [4].The channel codes are constructed based on the classical mathematical framework [8] of orthogonal designs. Space time block codes, which are designed in this way, exist for few sporadic values of $n$ transmit antennas and therefore a new mathematical theory was applied [7]. For complex signal constellations such as quadrature amplitude modulation (QAM), STBCs are designed to achieve the maximum possible transmission rate for any number of transmit antennas [6].

STBC technique is a special form of PHY diversity, which is a complex combination of coding theory, matrix algebra and signal processing [4]. It is a technique, which operates on a block of input symbols producing a matrix and outputs whose columns and rows represent time and antennas, respectively. A key feature of STBCs is the provision of full diversity with extremely low encoder/decoder complexity [5]. Therefore, STBCs can be effectively used to exploit the advantage of MIMO systems. With STBCs, the channel state information (CSI) is suggested to be available at the receiver and is practically calculated by the use of training symbols and pilot tones.

Hence, the most upcoming IEEE 802.11n standard proposals proposed to combine MIMO signal processing with OFDM in the next generation WLAN system. The target is to provide high data rates up to $540 \mathrm{Mbit} / \mathrm{s}$. It is known that the combination of MIMO and OFDM is a promising solution for enhancing the performance. High throughput in wireless communication systems can be achieved by increasing the number of antennas and doubling the bandwidth range. Furthermore, STBC techniques are also suggested in the MIMO-OFDM based 802.11n standard proposals by considering that STBC can help to achieve the 
full diversity and hence increase the data rates and throughput of an 802.11 n system.

The purpose of this paper is to evaluate the performance of 802.11n WWISE proposal [9] under a selection of different STBC rates. The system under investigation is a $4 \times 4$ MIMOOFDM PHY layer with 64-QAM modulation using Rayleigh frequency-selective fading and flat fading channels with STBC code rates 1 and $1 / 2$. Comparison is performed with a $2 \times 2$ MIMO-OFDM system in order to show full diversity performance of the system. Previous work provided us with results from some simple modulation techniques, e.g. QPSK modulation for the code rate of 1 and 16-QAM for the code rate of $1 / 2$ [11]. The scope of this paper is to advance the 802.11 performance on 64-QAM modulation technique with STBC code rates 1 and $1 / 2$, and to evaluate its performance through extensive simulations. The simulation results confirm that a significant performance gain can be achieved with a system of $1 / 2$ STBC code rate and multiple transmit/receive antennas, in comparison to those systems with STBC code rate 1.

The outline of this paper is organized as follows. Section II introduces the system model. In Section III, the numerical results obtained from the simulations are presented. Finally, Section IV draws the conclusions.

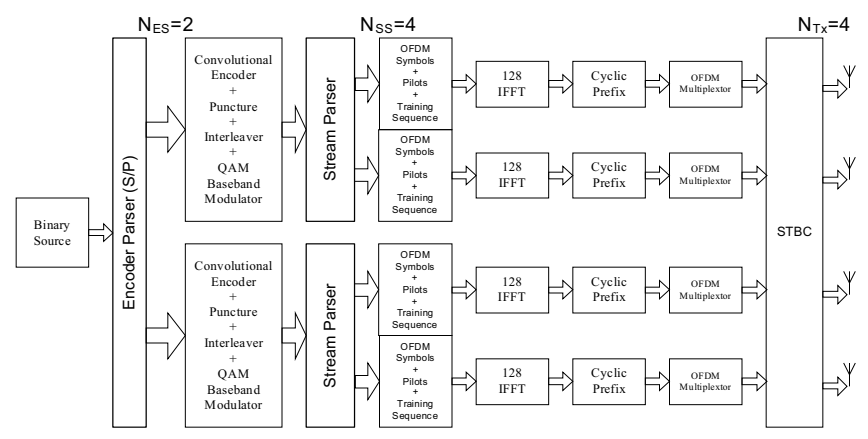

Fig. 1: MIMO-OFDM PHY layer model at transmitter

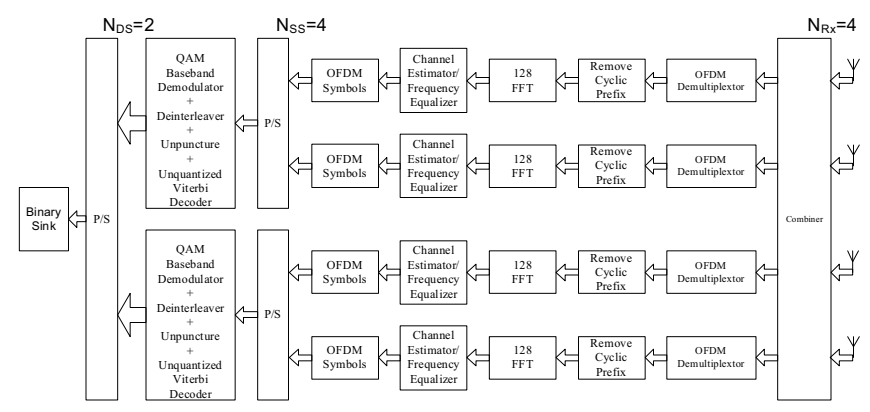

Fig. 2: MIMO-OFDM PHY layer model at receiver

\section{SYSTEM MODEL}

Recent developments focusing on the next-generation WLANs are proposed by several $802.11 \mathrm{n}$ proposals, e.g. TGnSync [13], WWISE [9] and MITMOT. These groups have recently agreed in submitting a unified proposal [12] to the IEEE 802.11n task group. The above proposals are based on the MIMO-OFDM technique for obtaining higher data throughput using multiple antenna arrays. The MIMO-
OFDM system considered in this paper closely resembles the 802.11n WWISE proposal in [9]. The channel bandwidths used are $20 \mathrm{MHz}$ and $40 \mathrm{MHz}$. Additionally, the 802.11n proposals include spatial multiplexing techniques in order to convert serial data stream to multiple parallel streams. In the following, we first give a brief overview of the $802.11 \mathrm{n}$ WWISE proposal [9]. Then both system and simulation models are presented based on the WWISE proposal framework.

\section{A. Model Description of $802.11 n$ PHY}

In 802.11n PHY layer, at the transmitter side a convolutional encoder is chosen to encode the binary source data, see Fig. 1. An encoder parser is a serial-to-parallel block that divides the original data stream fed from the source. After the encoder parser, the original data stream is converted to $N_{E S}(=2)$ parallel binary random data streams which then pass through another $N_{E S}$ encoders comprising convolutional encoder, puncturer, interleaver and modulator. A puncturer block has been added in order to achieve the code rates before the interleaver. The interleaver block is fed with coded bits from the convolutional encoder prior to a rectangular QAM baseband modulator using the Gray code for mapping the symbols. The stream parser module divides the output of the encoded stream into $N_{S S}$ spatial streams by assigning each frame for feeding the OFDM multiplexing and then the inverse fast Fourier transform (IFFT) process converts the frequency symbols into the time domain to the respective transmit antenna. A cyclic prefix has been added to avoid the ISI interference. Using the STBC code, the data passes to the receiver through Rayleigh (frequency-selective and flat fading) channel using $4 \times 4$ antennas. The process of the transmitted signal at the receiver passes via the next blocks in order to receive the appropriate data. On the receiver PHY layer system model (see Fig. 2), the OFDM frames are demultiplexed. The cyclic prefix that has been added to the transmitter is now removed. The FFT reconverts the process to the original state from time domain to the frequency domain. The training and pilot synchronization signals are removed whereas a frequency domain equalizer and a minimum mean square error (MMSE) channel estimator have been added. The data passes through the demodulator, deinterleaver, unpuncturer and Viterbi decoder. The decoder stream, $N_{D S}$, combines the decoded bits and the information data reach the sink.

\section{B. Simulation Overview}

As proposed in the 802.11n draft [9], the bandwidth of the OFDM signal is $40 \mathrm{MHz}$. In this case, the number of samples (Ns) is 128 for an OFDM data symbol which are equal to the number of subcarriers. The information-carrying data are encoded in the following modulation procedure. The binary source data sequence is first scrambled and then convolutionally encoded by the industrial standard rate $1 / 2$ encoder. The encoded output is then punctured according to the data rate requirement and is segmented into blocks of length $N_{C B P S}$, Table I, each of which corresponds to an OFDM data symbol. The binary data in each block is first interleaved among the subcarriers and then the information 
sequence is mapped in groups of bits into QAM symbols, which are used to modulate the different data carrying subcarriers. Each OFDM data symbol in the OFDM data field employs Ns $=128$ subcarriers, 108 of which are used for data symbols and 4 for pilot symbols and 1 DC (Direct Current). There are also 15 null subcarriers with 1 in the center and the other 14 on the two ends of the frequency band. The OFDM data symbols, each of which consists of $N s$ $=128$ samples, obtained via taking the IFFT of the data symbols, pilot symbols and nulls on these $N s$ subcarriers. To eliminate the ISI, each OFDM data symbol is preceded by a guard interval (GI), which contains the last samples of the data OFDM symbol [9].

\section{Channel description of the model}

So far, the transmission of the data stream with four transmit and four receive antennas has been described. In brief the sequence of the transmitter, the output bits after the encoder and especially from the interleaver of a single spatial stream are mapped to the subcarriers of the first antenna. Pair of complex numbers are transmitted through the four transmit antennas. The encoding scheme that has been used for this transmission is referred to as STBC. The STBC operates on a block of input symbols that produce a matrix output whose columns represent the time and rows of the antennas.

Hence, the output complex baseband signal $x_{i}^{k}(t)$ is filtered and converted to a radio frequency (RF) signal with a carrier frequency $f_{c}^{k}$.

$$
x_{i}^{k}(t)=\sqrt{\frac{E}{N_{T x}}} \sum_{n=0}^{N-1} d_{i}^{k}(n) \cdot \exp \left\{j \omega\left(t-T_{G I}\right)\right\},
$$

for $\mathrm{i}=1,2, \ldots, \mathrm{N}_{\mathrm{Tx}}$ transmit antennas and $\mathrm{k}=1,2, \ldots, \mathrm{K}$ OFDM blocks, where $\mathrm{E}$ is the average energy per transmitted symbol which is independent of the number of transmit antennas. $d_{i}^{k}(n)$ is the complex signal that is transmitted through the $n$-th subcarrier via the $i$-th transmit antenna during the $k$-th OFDM period. $\omega=2 \pi /\left(N T_{s}\right)$ is the frequency separation between two adjacent subcarriers. $T_{G I}$ is the guard interval duration with $T_{G I}=N T_{s} / 4$. Therefore, the signal operates in the interval of $\left[T_{G I}, T_{G I}+N T_{s}\right]$.

The Rayleigh frequency-selective fading and flat fading channels are chosen such that the signal can be highly distorted and distinct, respectively [10]. As mentioned earlier, by using CSI, the channel is unknown to the transmitter but is known to the receiver. The received data stream $r_{j}^{k}(n)$ at the $n$-th subcarrier and the $j$-th receive antenna during $k$-th OFDM symbol duration with Additive
White Gaussian Noise (AWGN), $w_{j}^{k}(n)$, can be expressed by the following equation:

$$
r_{j}^{k}(n)=\sqrt{\frac{E}{N_{T x}}} \sum_{n=0}^{N_{T x}-1} H_{i j}^{k}(n) \cdot d_{i}^{k}(n)+w_{j}^{k}(n)
$$

$H_{i j}^{k}(n)$ is the frequency response of the channel

$$
H_{i j}^{k}(n)=\sum_{l=0}^{L-1} a_{i j}^{k}(l) \cdot \exp \left\{-j n 2 \pi f \tau_{l}\right\}
$$

and where $a_{i j}^{k}(l)$ is the multipath gain coefficient for $L$ available paths and $\tau_{l}$ is the path delay of the $l$-th path.

\section{NumericAl Results}

The main purpose of the simulation is to analyze the performance of the $802.11 \mathrm{n}$ for various data rates under different STBC code rates with 64-QAM modulation using Rayleigh channels. In our simulations, we set the transmit antennas $\mathrm{N}_{\mathrm{Tx}}$ and the receive antennas $\mathrm{N}_{\mathrm{Rx}}$ for the $4 \mathrm{x} 4$ system as $\mathrm{N}_{\mathrm{Tx}}=\mathrm{N}_{\mathrm{Rx}}=\mathrm{N}_{\mathrm{SS}}=4$ (where $\mathrm{N}_{\mathrm{SS}}$ is the number of spatial streams) as illustrated in Fig. 1 with encoded streams $\mathrm{N}_{\mathrm{ES}}=2$ and decoded streams $\mathrm{N}_{\mathrm{DS}}=2$ in Fig. 2. Further a comparison is performed between 1/2 STBC 4x4 MIMOOFDM system and a $2 \times 2$ system under STBC code rate 1 using $\mathrm{N}_{\mathrm{ES}}=1$ and $\mathrm{N}_{\mathrm{DS}}=1$ via a Rayleigh flat-fading channel.

The specifications that are used for the simulation setup for the $4 \times 4$ system are presented in Table I. For the $2 \times 2$ system half packet length size is applied for each data rate. The transmitter sends 16 MIMO-OFDM data symbols per spatial stream. Different packet lengths have been transmitted for different data rates. To obtain the average

\begin{tabular}{|c|c|c|c|c|c|c|c|}
\hline $\begin{array}{l}\text { Data } \\
\text { rate } \\
\text { (Mbps) }\end{array}$ & Modulation & $\begin{array}{l}\text { Code } \\
\text { rate } \\
\text { (R) }\end{array}$ & $\begin{array}{c}\text { Number of } \\
\text { data } \\
\text { subcartiers } \\
\left(N_{y}\right)\end{array}$ & $\begin{array}{l}\text { Number } \\
\text { of } \\
\text { pilots } \\
\left(\mathrm{N}_{\mathrm{s}}\right)\end{array}$ & $\begin{array}{l}\text { Coded bits } \\
\text { MIMO-OFDM } \\
\text { symbol } \\
\left(\mathrm{N}_{\text {OBW }}\right)\end{array}$ & $\begin{array}{c}\text { Databits } \\
\text { MIMO-OFDM } \\
\text { symbol } \\
\text { (Nom) }\end{array}$ & \\
\hline 432 & 64.0 $\mathrm{AM}$ & $2 B$ & 108 & 4 & 2592 & 1728 & \\
\hline 486 & $64.0 \mathrm{AM}$ & $3 / 4$ & 108 & 4 & 2592 & 1944 & \\
\hline 540 & 64.QAM & 56 & 108 & 4 & 2592 & 2160 & \\
\hline
\end{tabular}
performance, 1000 packets are transmitted for each data rate.

TABLE I

From Fig. 3 and Fig. 4 it can be observed that the maximum data rate is $540 \mathrm{Mbit} / \mathrm{s}$ for $4 \times 4$ system at $20 \mathrm{~dB}$ and $26 \mathrm{~dB}$ using STBC code rate 1 under Rayleigh flat fading and frequency-selective fading channel, respectively. For $1 / 2$ STBC code rate the system also achieves the highest data rate that gains approximately $4 \mathrm{dBs}$ for Rayleigh flat 
fading channel and 6 to $7 \mathrm{~dB}$ for Rayleigh frequencyselective channels. The simulation results shown in Fig. 3 are an average over ten experiments for each data rate. However, comparing Fig. 3 and Fig. 4, it is obvious that the Rayleigh frequency-selective fading channel requires more power in order to achieve the appropriate data rate than the Rayleigh flat fading channel. The signal-to-noise ratio (SNR) varies from 3 to $4 \mathrm{dBs}$ that actually means duplication of the signal power. The highest data rate $(540 \mathrm{Mbit} / \mathrm{s})$ is achieved in our system that is ten times more according to the existing 802.11a protocol. Performance gain is achieved 4 to $7 \mathrm{dBs}$ less depending on the channel selection with $1 / 2$ STBC code rate under 64-QAM modulation.

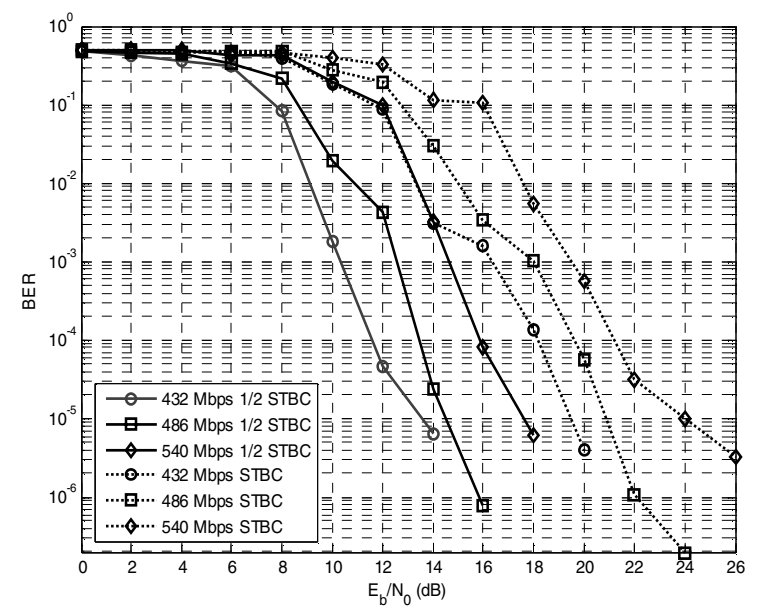

Fig. 3: BER simulation results with 1 and $1 / 2$ STBC under Rayleigh frequency-selective fading channel

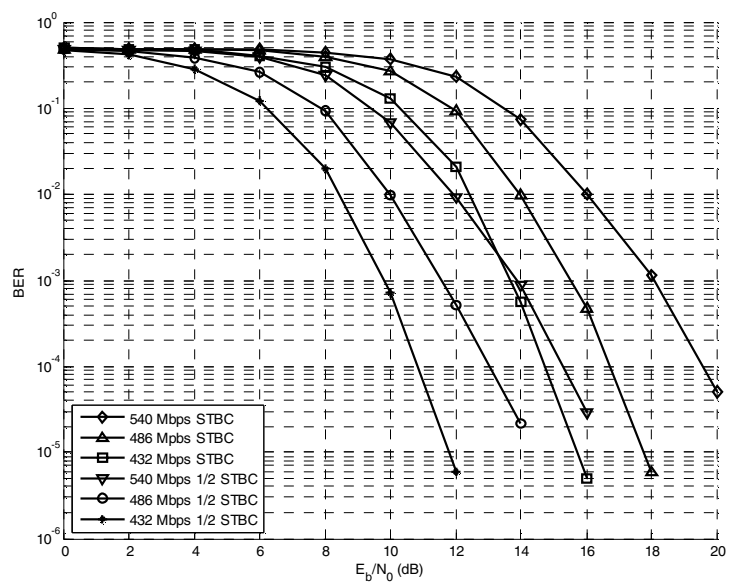

Fig. 4: BER simulation results with 1 and $1 / 2$ STBC under Rayleigh flat-fading channel

Also, from Fig. 4 the maximum data rate is $540 \mathrm{Mbit} / \mathrm{s}$ for $4 \times 4$ system at $20 \mathrm{~dB}$ using STBC code rate 1 under Rayleigh flat fading channel. For $1 / 2$ STBC code rate the system is also setup to operate in the maximum data rate and shows a gain in performance of approximately $4 \mathrm{dBs}$. This performance gain is due to the transmitted replicas of the signal which are provided in the form of redundancy in the time domain. So there is diversity in time.
In Fig. 5 a comparison is presented between two different systems. These are the above designed $4 \mathrm{x} 4$ MIMO-OFDM system using 1/2 STBC code rate and a $2 \times 2$ MIMO-OFDM system using STBC code rate 1, both using the 64-QAM technique, under a Rayleigh flat-fading channel. Simulation results show that the $4 \times 4$ system with $1 / 2$ STBC code rate has a gain of $7 \mathrm{dBs}$ comparing to the $2 \times 2$ system with STBC code rate 1 at all data rates. This performance gain is due to the transmitted replicas of the signal and due to the antenna diversity of the system. More antennas give higher diversity and therefore better estimation at the receiver for the transmitted signal.

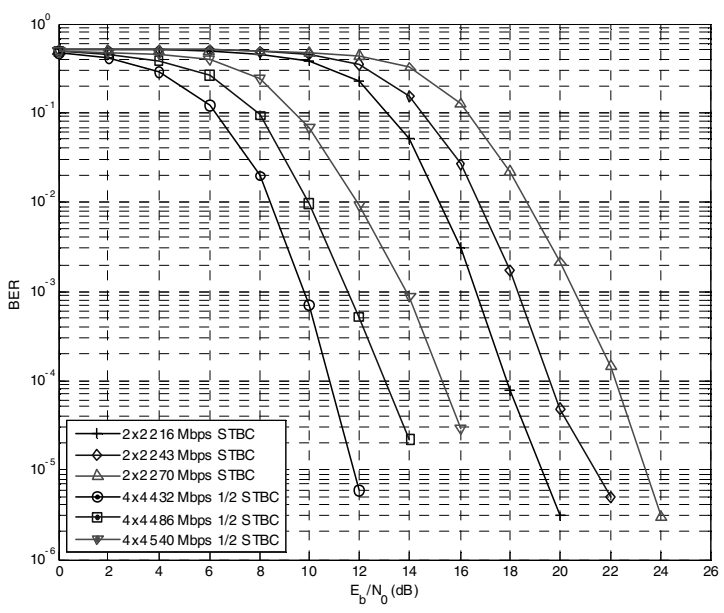

Fig. 5: BER simulation results with $2 \times 2$ using STBC 1 and $4 \times 4$ using $1 / 2$ STBC under Rayleigh flat-fading channel

\section{CONCLUSION}

This paper presented the performance analysis of the upcoming 802.11n PHY layer model based on the 802.11n WWISE proposal [9] with four transmit and four receive antennas by comparing it under different STBC rates as well as with $2 \times 2$ antenna system. Our simulation model can also be easily extended to study other $802.11 \mathrm{n}$ proposals. We provide two examples of space-time block codes using multiple transmit and multiple receive antennas. Simulation results were carried out using Rayleigh frequency-selective fading and flat fading channel with 64-QAM modulation technique under different STBC code rates. It is evident from the numerical results that the physical layer model with four transmit and four receive antennas and 1/2 STBC code rate offers higher diversity in time and results a gain in performance. Furthermore simulations show that the $4 \times 4$ with $1 / 2$ STBC model has a performance gain when it is compared with a $2 \times 2$ model using STBC rate 1 . This comparison was made in order to show the full diversity performance of the system.

\section{REFERENCES}

[1] R. S. Blum, Y. Li, J. H. Winters, Q. Yan, "Improved Space-Time Coding for MIMO-OFDM Wireless Communications," IEEE Trans. Commun., pp. 1873-1878, Nov. 2001. 
[2] D. Gebert, M. Shafi, D. Shiou, P. J. Smith, A. Naguib, "From Theory to Practice: An Overview of MIMO Space-Time Coded Wireless Systems," IEEE J. Select Areas Commun., pp. 281-302, Apr. 2003.

[3] V. Tarokh, N. Seshadri, A. R. Calderbank, "Space-Time Codes for High Data Rate Wireless Communications: Performance Criterion and Code Construction," IEEE Trans. Inform. Theory, vol. 44, No 2, pp.744-765, Mar. 1998.

[4] A. Stamoulis, N. Al-Dhahir, "Impact of Space-Time Block Codes on 802.11 Network Throughput," IEEE Trans. Wirel. Commun., vol.2, No 5, pp. 1029-1039, Sept. 2003.

[5] S. Sandhu, R. Heath, A. Paulraj, "Space-Time Codes Versus SpaceTime Trellis Codes,"IEEE Proc. of IEEE International Conf. on Communications, pp. 1132-1136, Helsinki, Finland, June 11 - 14, 2001.

[6] V. Tarokh, H. Jafarkhani, A. R. Calderbank, "Space-Time Block Coding for Wireless Communications: Performance Results,"'IEEE Jour.on selected Areas in Commun. ,vol.17, No 3, Mar. 1999.

[7] V. Tarokh, H. Jafarkhani, A. R. Calderbank, "Space-Time Block Codes from Orthogonal Designs," IEEE Trans. on Inform. Theory, Vol 45, No 5, July 1999.

[8] V. Tarokh, H. Jafarkhani, A. R. Calderbank, "The application of orthogonal designs to wireless communicaiton," IEEE Inform. Theory Workshop, Killarney, Ireland, pp.46-47, June 1998.

[9] WWISE proposal: High throughput extension to $802.11 \mathrm{n}$ standard http://www.wwise.org/.

[10] E. Biglieri, J. Proakis, S. Shamai, "Fading Channels: InformationTheoretic and Communication Aspects," IEEE Trans. Inform. Theory, vol. 44, No 6, pp.2619-2692, Oct. 1998.

[11] H. Jafarkhani, “A Quasi-Orthogonal Space-Time Block Code," IEEE Trans. on Commun., Vol. 49, No 1, Jan. 2001.

[12] IEEE Draft amendment to standard: Enhancements for Higher Throughput, IEEE P802.11n /D1.0, March 2006.

[13] TGn Sync proposal: doc.: IEEE 802.11-04/0889r3. 